\title{
Pulsating, Creeping, and Erupting Solitons in Dissipative Systems
}

\author{
J. M. Soto-Crespo, ${ }^{1}$ N. Akhmediev, ${ }^{2}$ and A. Ankiewicz ${ }^{2}$ \\ ${ }^{1}$ Instituto de Óptica, C.S.I.C., Serrano 121, 28006 Madrid, Spain \\ ${ }^{2}$ Optical Sciences Centre, Research School of Physical Sciences and Engineering, Australian National University, \\ Australian Capital Territory 0200, Australia
}

(Received 5 May 2000)

\begin{abstract}
We present three novel pulsating solutions of the cubic-quintic complex Ginzburg-Landau equation. They describe some complicated pulsating behavior of solitons in dissipative systems. We study their main features and the regions of parameter space where they exist.
\end{abstract}

PACS numbers: 42.65.Tg, 05.45.Yv, 05.70.Ln, 47.20.Ky

A soliton is a self-localized solution of a nonlinear partial differential equation describing the evolution of a nonlinear dynamical system with an infinite number of degrees of freedom. Solitons are usually attributed to integrable systems. In this instance, solitons remain unchanged during interactions, apart from a phase shift. They can be viewed as "modes" of the system, and, along with radiation modes, they can be used to solve initial value problems, using a nonlinear superposition of modes [1]. The two main features of solitons in integrable systems which are of interest to us are, first, that they are one- (or few-)parameter families and, second, that the superposition of solitons with zero velocity produces a pulsating solution [2], which is sometimes called a "breather."

Reductions to integrable systems are extreme simplifications of the complex systems existing in nature. They can be considered as a subclass of the more general Hamiltonian systems [3]. Indeed, such a simplification allows us to analyze the systems quantitatively and to completely understand the behavior of the solitons. Solitons in Hamiltonian (but nonintegrable) systems can also be regarded as nonlinear modes, but in the sense that they allow us to describe the behavior of systems with an infinite number of degrees of freedom in terms of a few variables, thus allowing us to effectively reduce the number of degrees of freedom. Solitons in these systems collide inelastically and interact with radiation waves, thus showing that they are qualitatively different from those in integrable systems. However, as in the integrable case, the solitons are still a one- (or few-)parameter family of solutions. Another interesting property of Hamiltonian systems is that there are no pulsating solutions. If the system is near integrable, then the two-soliton solutions of the nonlinear Schrödinger equation (NLSE) which are initially excited will gradually split into two solitons or transform into a single soliton solution, depending on the type of the perturbation [4]. If the system is far from integrable, pulsations may exist if a single soliton solution is excited with a perturbation; however, they die out, so that the pulse gradually converges to a stationary soliton.

Dissipative systems are more complicated than Hamiltonian ones in the sense that, in addition to nonlinearity and dispersion, they include energy exchange with exter- nal sources. The generic equation which describes dissipative systems above the point of bifurcation is the complex Ginzburg-Landau equation (CGLE) [5,6]. A review of experiments described by the CGLE is given in [7]. In optics, it describes laser systems [8-11], soliton transmission lines [12], nonlinear cavities with external pump [13], and parametric oscillators [14]. Importantly enough, solitons, when they exist [15], can again be considered as modes of these systems, thus allowing us to describe the behavior of the system in terms of just a few degrees of freedom. However, there is a significant difference between solitons in Hamiltonian and dissipative systems. In Hamiltonian systems, soliton solutions appear as a result of a balance between diffraction (dispersion) and nonlinearity. Diffraction spreads the beam while nonlinearity focuses it and makes it narrower. The balance between the two results in a stationary solution, which is usually a one-parameter family. In systems with gain and loss, in order to have stationary solutions, gain and loss must be also balanced. This additional balance results in solutions which are fixed. The shape, amplitude, and the width are all fixed and depend on the parameters of the equation [16]. There are some exceptions to this rule $[17,18]$ but these are very special cases. There are four known analytic solutions of the cubicquintic CGLE: solitons, kinks, sources, and sinks [19]. If not limited to analytic solutions, the variety of stationary localized structures can be much higher [3].

Dissipative systems, in contrast to Hamiltonian ones, admit pulsating solutions. However, they do not appear from the integrable limit and, hence, do not have anything in common with the nonlinear superposition of fundamental solitons of the NLSE [2]. The parameters of the CGLE have to be far enough from the NLSE limit in order to obtain pulsating solitons. An example of a pulsating soliton of the cubic-quintic CGLE in the region of normal dispersion has been presented by Deissler and Brand [20]. This solution has been found in the normal dispersion regime, where solitons do not exist in the integrable limit. In fact, this is not the only case where pulsating solutions exist. They do exist in the anomalous dispersion region and, moreover, the variety of these solutions and their region of existence is huge. Nevertheless, even in this case, pulsating solutions cannot be found by extrapolating from those in the integrable limit. 
In this work we report the discovery of three new types of cubic-quintic CGLE robust pulsating soliton solutions with complicated behavior. They exist in three isolated regions of the parameter space, a fact which facilitates their identification as three different solutions. All these solutions have two common features - they repeat periodically in the $z$ direction (the propagation direction) and they are actually pulsating. We should say that there can be a great variety of pulsating structures. All the solutions we have found have distinctive features, and this allows us to classify them as "plain pulsating," "erupting," or "creeping" solitons. We have studied their main characteristics and investigated in detail the region in the parameter space where they exist. None of them can be found in analytic form, and this feature is similar to stable stationary solitons of the cubic-quintic CGLE [3]. However, pulsating solutions are generic in the sense that they occupy appreciable regions of the five-dimensional parameter space. Besides, they can be excited from a wide range of initial conditions. Eventually, and usually very quickly, each of them will converge to that pulsating soliton which exists for the given set of the equation parameters. An exception to this rule occurs when two or more solutions exist for the same set of parameters. When broad (but still localized) initial conditions are used, several pulsating solitons can be excited simultaneously.

The $(1+1)$ dimensional cubic-quintic CGLE describes situations where there is a single transverse (or temporal) coordinate (see, e.g., $[3,16])$ :

$$
\begin{aligned}
i \psi_{z}+\frac{D}{2} \psi_{\tau \tau}+|\psi|^{2} \psi= & i \delta \psi+i \epsilon|\psi|^{2} \psi+i \beta \psi_{\tau \tau} \\
& +i \mu|\psi|^{4} \psi-\nu|\psi|^{4} \psi
\end{aligned}
$$

where $\delta, \beta, \epsilon, \mu, D$, and $\nu$ are real constants, and $\psi$ is a complex field. The physical meaning of each variable depends on the particular problem. In optics, $\tau$ is the retarded time (or a transversal spatial coordinate), $z$ is the propagation distance, and $D$ represents dispersion (or diffraction). By a proper rescaling in $\tau, D$ can be fixed to take the values of \pm 1 , without loss of generality. Hence, the number of independent parameters in Eq. (1) is five.

An example of a pulsating soliton found numerically is shown in Fig. 1. It shows perfectly periodic behavior with the period in $z$ being around 14. It has a different shape at each $z$, since it evolves, but it recovers its exact initial shape after a period. In this sense, we can call this type a "plain" pulsating soliton. The solutions found by Deissler and Brand [20] do not belong to this class, because, in their case, the dispersion parameter $D$ has the opposite sign, so that the region of parameters where they exist is different. It is also noteworthy that, contrary to the cases reported here, the profile of the periodic solutions in Ref. [20] changes only in the soliton tails. It also keeps its value of energy almost constant, while, in the case of the solution shown in Fig. 1, the energy, $Q=\int_{-\infty}^{\infty}|\psi|^{2} d \tau$, changes from about 10 to 42 (Fig. 2). When we change the parameters of

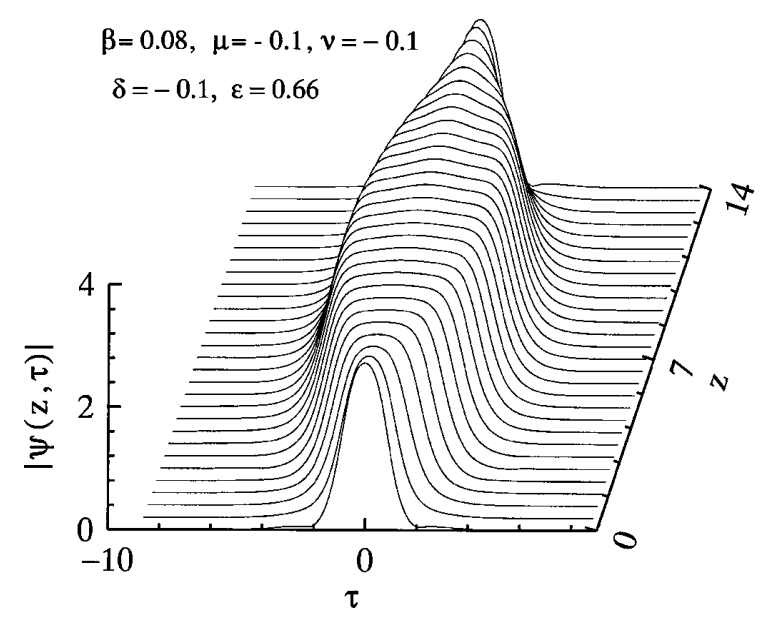

FIG. 1. Plain pulsating soliton of CGLE. The parameters are $D=+1, \epsilon=0.66, \delta=-0.1, \beta=0.08, \mu=-0.1$, and $\nu=-0.1$.

the equation, the solution remains pulsating in a certain region which does not extend to the region with negative $D$.

A sample of the region (shaded) in the parameter space with pulsating solutions is shown in Fig. 3. Beyond this region, the solution either transforms into a stationary solution (soliton or front) or bursts into chaos. The darkest areas in Fig. 3, labeled II and IV, show the regions where period-2 and period-4 pulsating solutions were found. This fact indicates that the route to chaos in this particular case is through period doubling bifurcations. However, in general, the route to chaos can vary at the edges of the region and is a complicated topic to study because we have five parameters to change. In other cases (not shown here), the pulsating solution can also be quasiperiodic, with several incommensurate periods involved in the evolution. The energy, $Q$, versus $z$ for these solutions is also quasiperiodic, with several periods involved. In both cases, purely periodic or quasiperiodic pulsating continues indefinitely in $z$.

Another class of pulsating solitons can be called erupting solitons. Erupting soliton evolution (see Fig. 4) starts from a stationary localized solution which has a perfect soliton shape. After a while its "slopes" become covered with small ripples (small scale instability) which seem to move downwards along the two slopes of the soliton, and very soon the pulse is covered with this seemingly

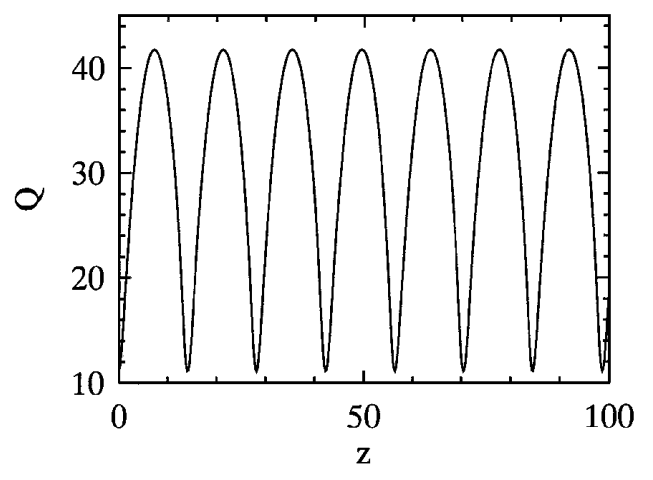

FIG. 2. Energy, $Q$, versus $z$ for the solution in Fig. 1 . 


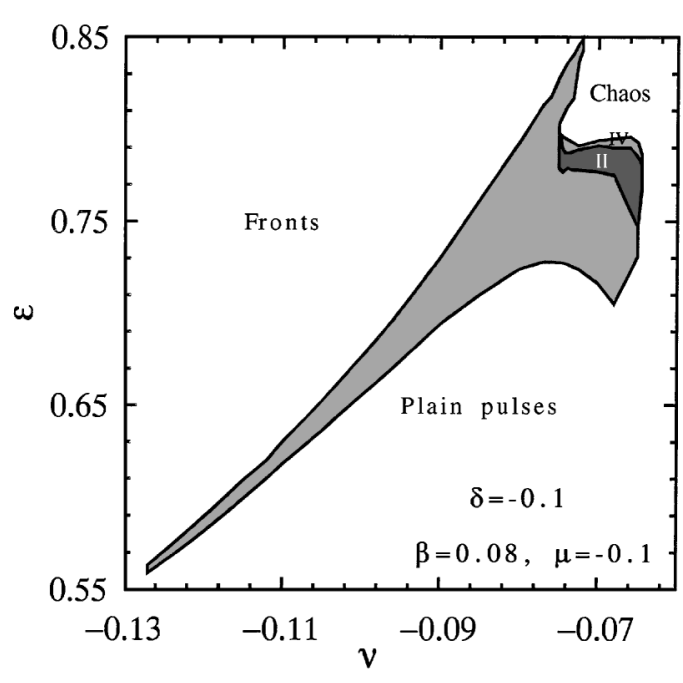

FIG. 3. Region in the parameter plane $(\nu, \epsilon)$ where pulsating localized solutions exist. The values of other parameters are $D=+1, \delta=-0.1, \beta=0.08$, and $\mu=-0.1$.

chaotic structure. When the ripples increase in size, the soliton cracks into pieces, like a mountain after a strong volcanic eruption or after an earthquake. This completely chaotic, but well-localized, structure then is filled with "lava" which restores the perfect soliton shape after a "cooling" process. The process repeats forever, although the distance between "eruptions" fluctuates, and in each of them the pulse splits into different pieces. Needless to say, these solutions cannot be found in analytic form. However, they are as common as stationary solutions and exist for a wide range of parameters, as seen in Fig. 5.

The process never repeats itself exactly in successive "periods." However, it always returns to the same shape. In this sense, the orbit which corresponds to this solution in each period is homoclinic. Figure 6 shows the spectral width, $\sigma_{F}=\sqrt{\left\langle f^{2}\right\rangle-\langle f\rangle^{2}}$, where $f$ is the frequency, versus temporal width, $\sigma_{T}=\sqrt{\left\langle\tau^{2}\right\rangle-\langle\tau\rangle^{2}}$ of the erupting soliton during several successive "periods" of eruption.

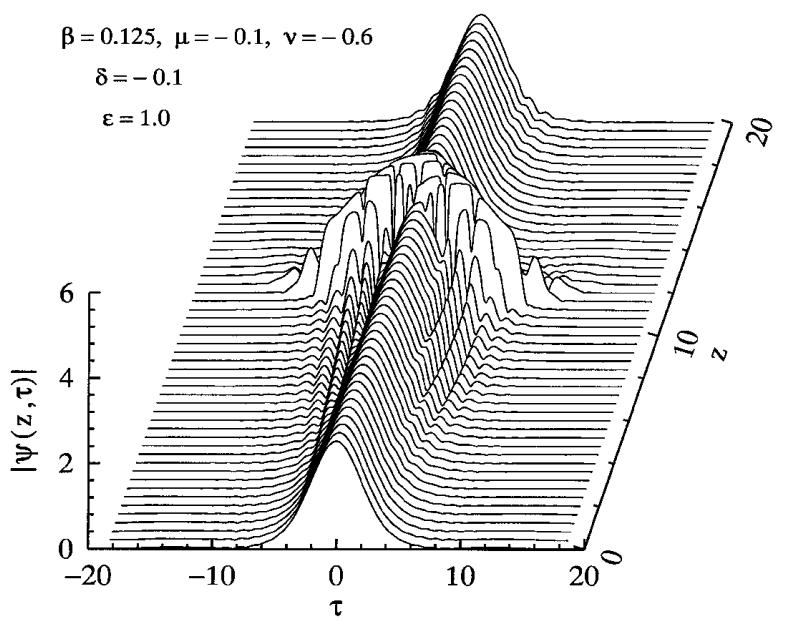

FIG. 4. Erupting soliton of the quintic CGLE. The parameters are $\epsilon=1.0, \delta=-0.1, \beta=0.125, \mu=-0.1$, and $\nu=-0.6$.
Here, $\left\langle\tau^{n}\right\rangle$ stands for $\int_{-\infty}^{\infty} \tau^{n}|\psi|^{2} d \tau / Q$, and the same applies for $f$ in the spectral domain. Although each part of the total trajectory starts and ends at the same point, which corresponds to the solution in the quiet part of the evolution, where it changes only slightly, they are all different during the erupting stage of the evolution. This shows that the evolution never repeats itself and that each eruption is unique. Hence, the quiet stage of the soliton is an attractor, but is an unstable one. The length of each period also varies slightly, as it should for an attractor. The average position of the pulse shifts in $\tau$.

As seen from Fig. 6, the product $\sigma_{F} \sigma_{T}$ is around 1. This is more than 10 times greater than the same product for bandwidth-limited pulses, which is $1 / 4 \pi \approx 0.08$. This shows that the soliton is highly chirped and literally "tries" to split apart during the quiet regime of evolution. It is remarkable that the spectrum of the solitons becomes narrower during the burst (from $\sigma_{F} \approx 0.64$ down to $\sigma_{F} \approx 0.51$ ). However, the product $\sigma_{F} \sigma_{T}$ increases during the eruption due to the chaotic structure of the solution in time. This product returns to its previous value when a new soliton emerges from the fragments of the burst. The growth rate of instability is actually complex and is equal to $0.8+16.7 i$ for this particular case. The imaginary part of the growth rate eigenvalue is responsible for the radiative structure around the soliton. The total energy in the soliton, $Q$, also pulsates and increases during the burst by almost a factor of 5 from its value in the quiet regime ( $Q=22)$ up to $Q=100$.

Figure 5 shows the region of existence of erupting solitons in the $(\nu, \epsilon)$ plane. The strip where these solutions exist is relatively wide, so these solutions cannot be "missed." In the lower limit in $\epsilon$, these solutions either become periodic solutions or are extinguished completely. In the upper limit, above the shaded strip, they become either chaotic or stationary pulses.

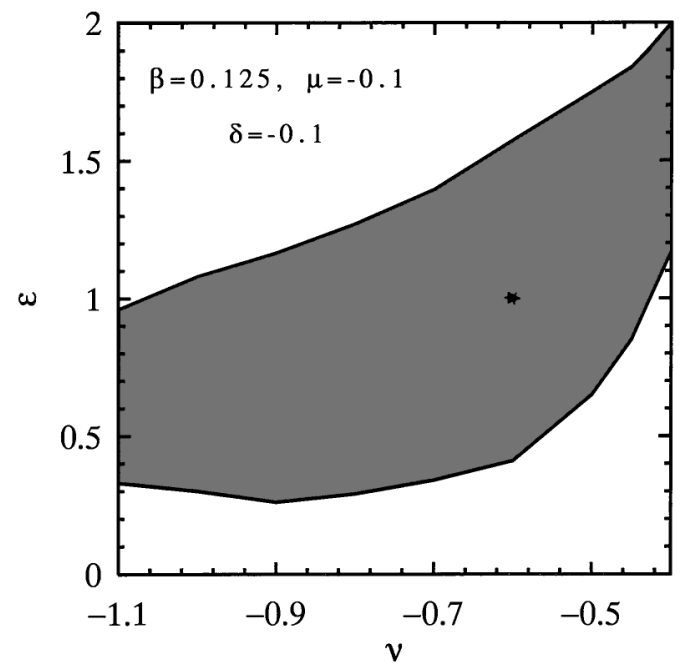

FIG. 5. Region in the parameter plane $(\nu, \epsilon)$ where erupting solitons exist. The star shows the point where the simulations for Fig. 4 were done. Other parameters are $\delta=-0.1, \beta=0.125$, and $\mu=-0.1$. 


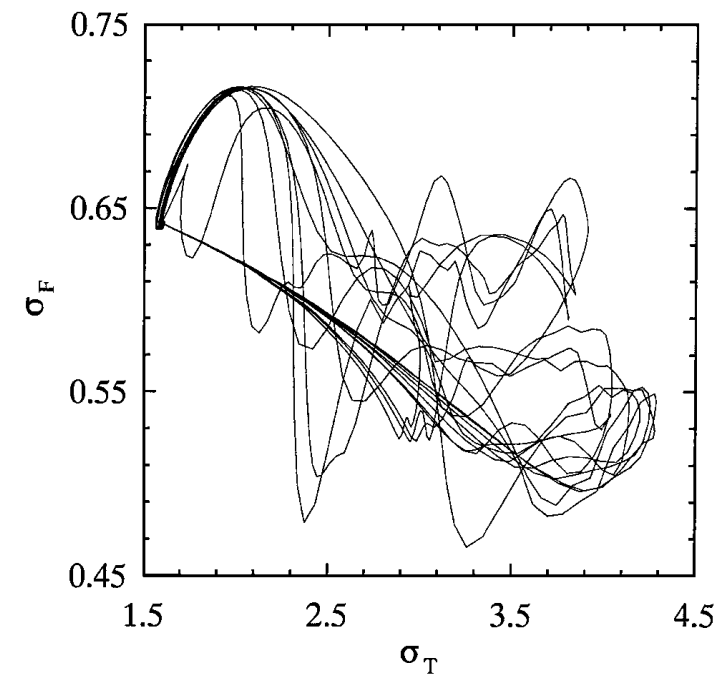

FIG. 6. The spectral width versus spatial width for the evolution of erupting solitons during successive cycles. The parameters are the same as in Fig. 4.

Our final example is the creeping soliton which is shown in Fig. 7. It is a rectangular pulse with two fronts and a sink (due to energy loss) at the top. The two fronts pulsate back and forth relative to the sink asymmetrically at the two sides. As a result of this asymmetry, the position of the center of mass of the whole soliton shifts after each pulsation. The accumulated shifts result in soliton motion with constant velocity. This pulse coexists with the soliton with two fronts which are symmetrically pulsating on each side. The shape of the creeping soliton resembles the shape of the composite soliton [3]. In contrast to the two previous examples, these solutions exist at the boundary between solitons and fronts [21]. This region is isolated from the region of erupting solitons. Again, these can be classified as a separate type of soliton.

We have to note that the variety of localized pulsating solutions found here results from the properties of the cubicquintic CGLE. A system has to have a certain minimum complexity in its nonlinear properties in order to have $l o-$ calized robust pulsating solutions. The nonlinear terms in the case of the cubic CGLE are much simpler and, as a re-

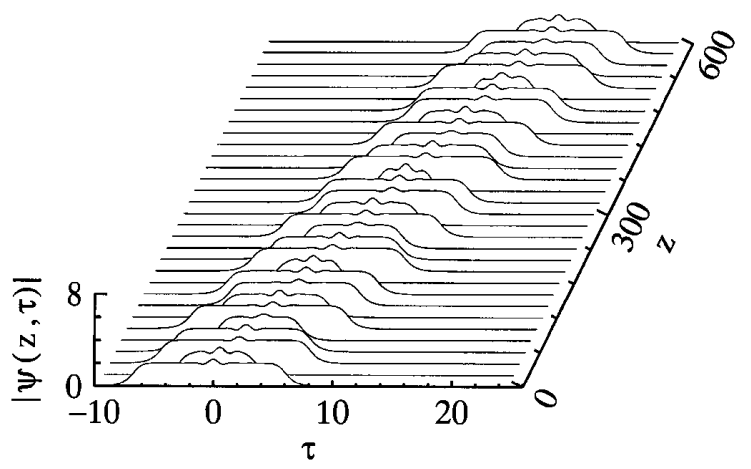

FIG. 7. Creeping soliton of the quintic CGLE. The parameters are $D=+1, \epsilon=1.3, \delta=-0.1, \beta=0.101, \mu=-0.3$, and $\nu=-0.101$. sult, the cubic CGLE in the $(1+1) \mathrm{D}$ case does not have such solutions. We recall, in this respect, that even stationary solitons of the cubic CGLE in general are not stable [3], and quintic terms are essential for stable solitons to appear [11]. We also have to note that, for the cubic-quintic CGLE, the solutions given here are only some examples of localized pulsating solitons. There is great variety of them and a great variety of regions in the parameter space where they exist. We think that our present work will serve as an ignition point for a further burst of study of this phenomenon, as it is interesting from both theoretical and experimental points of view.

In conclusion, we have found, numerically, three novel types of robust pulsating soliton solutions of the 1D complex cubic-quintic Ginzburg-Landau equation and analyzed their properties and regions of existence in parameter space.

The work of J.M. S. C. was supported by the Dirección General de Enseñanza Superior under Contract No. PB960819. N. A. and A. A. are members of the Australian Photonics Cooperative Research Centre (APCRC).

[1] V.E. Zakharov and A. B. Shabat, Sov. Phys. JETP 34, 62-69 (1971).

[2] J. Satsuma and N. Yajima, Prog. Theor. Phys. Suppl. 55, 284-306 (1974).

[3] N. N. Akhmediev and A. Ankiewicz, Solitons: Nonlinear Pulses and Beams (Chapman and Hall, London, 1997).

[4] D. Artigas, L. Torner, and N. N. Akhmediev, Opt. Commun. 143, 322 (1997).

[5] Y. Kuramoto, Chemical Oscillations, Waves and Turbulence (Springer-Verlag, Berlin, 1984).

[6] C. Normand and Y. Pomeau, Rev. Mod. Phys. 49, 581-623 (1977).

[7] M. C. Cross and P. C. Hohenberg, Rev. Mod. Phys. 65, 851 (1993).

[8] H. Haus, J. Appl. Phys. 46, 3049 (1975).

[9] P. A. Belanger, J. Opt. Soc. Am. B 8, 2077 (1991).

[10] C. O. Weiss, Phys. Rep. 219, 311-338 (1992).

[11] J. D. Moores, Opt. Commun. 96, 65 (1993).

[12] L. F. Mollenauer, J. P. Gordon, and S. G. Evangelides, Opt. Lett. 17, 1575 (1992).

[13] W. J. Firth and A.J. Scroggie, Phys. Rev. Lett. 76, 1623-1626 (1996).

[14] P.-S. Jian, W. E. Torruellas, M. Haelterman, S. Trillo, U. Peschel, and F. Lederer, Opt. Lett. 24, 400 (1999).

[15] S. Fauve and O. Thual, Phys. Rev. Lett. 64, 282 (1990).

[16] W. van Saarloos and P.C. Hohenberg, Physica (Amsterdam) 56D, 303 (1992).

[17] N. Bekki and K. Nozaki, Phys. Lett. 110A, 133 (1985).

[18] N. N. Akhmediev and V. V. Afanasjev, Phys. Rev. Lett. 75, 2320-2323 (1995).

[19] R. Conte and M. Musette, Physica (Amsterdam) 69D, $1-17$ (1993).

[20] R. J. Deissler and H. R. Brand, Phys. Rev. Lett. 72, 478-481 (1994).

[21] V. Hakim, P. Jakobsen, and Y. Pomeau, Europhys. Lett. 11, 19-24 (1990). 